\title{
Polos epistemológicos: uso y construcción de teoría en investigación cualitativa en educación
}

\section{Resumen}

En este artículo se proponen e ilustran los conceptos de polo epistemológico I (cercano a los marcos teóricos del investigador) y polo epistemológico II (cercano a la perspectiva de los participantes de una realidad educativa). Se plantea que el tránsito constante y permanente entre ambos polos y la puesta en marcha del mecanismo de vigilancia epistemológica permiten la construcción de conocimiento teórico en investigación cualitativa. Al finalizar, se presta especial atención al uso de la teoría durante las diversas etapas del proceso investigativo y las formas en que las teorizaciones iniciales pueden ser descartadas o ampliadas.

\section{Palabras clave descriptor}

Epistemología, teoría de la educación, análisis cualitativo.

\section{Transferencia a la práctica}

Este artículo se refiere a cuestiones epistemológicas que son clave para la construcción de nuevo conocimiento en el ámbito educativo. Propone y ejemplifica los conceptos de polo epistemológico | y || y expone el mecanismo de relevancia metodológica como cuestiones clave que ayudarán, tanto a investigadores jóvenes como consolidados, a reflexionar sobre el rol de los marcos ontoespistémicos e ideológicos en la construcción de nueva teoría. Asimismo, resalta la necesidad del uso crítico y reflexivo de teoría a lo largo del proceso investigativo, así como la importancia de aumentar, problematizar y contrastar el corpus teórico existente.

Para citar este artículo / To cite this article / Pour citer cet article / Para citar este artigo

Guzmán-Valenzuela, C. M. (2014). Polos epistemológicos, uso y construcción de teoría en investigación cualitativa en educación. magis, Revista Internacional de Investigación en Educación, 7(14), 15-28. 
Key words plus

Epistemology, Theory of Education, Qualitative Analysis.

\section{Abstract}

In this article the concepts of epistemological pole I (close to the theoretica frameworks of the researcher) and epistemological pole II (close to the participants perspective from an educational reality) are proposed and illustrated. It is argued between both poles, as well as the implementation of the epistemological vigilance mechanism enable the development of theoretical knowledge in qualitative research. The article concludes by paying particular attention to the use of theory during the various stages of the research process and the ways in which initial theories can be discarded or extended. that the constant and permanent transit

\section{Transference to practice}

This article addresses epistemological questions that are key to the construction of new knowledge in the educational field. It proposes and exemplifies the concepts of epistemological pole I and II, as well as the mechanism of methodological relevance, both issues are key to help both young researchers and seniors to reflect on the role played by onto-epistemic and ideological frameworks in the development of new theory. It also highlights the need for critical and reflective use of theory throughout the research process and the importance of increasing, problematizing and contrasting existing theoretical corpus.

\section{Résumé}

Dans cet article on expose et illustre les concepts du pôle épistémologique I (proche aux cadres théoriques du chercheur) et du pôle épistémologique II (proche à la perspective des participants d'une réalité éducative). On montre que le transit constant et permanant entre les deux pôles ainsi que la mise en marche du mécanisme de vigilance épistémologique permet la construction de la connaissance théorique dans la recherche qualitative. L'article finalise en portant spéciale attention à l'usage de la théorie pendant les différentes étapes du processus de la recherche et les formes comment les théorisations initiales peuvent être écartées ou amplifiées.

\section{Transfert à la pratique}

Cet article fait référence aux questions épistémologiques clés pour la construction des nouvelles connaissances dans le domaine éducative. II propose et illustre les concepts du pôle épistémologique I et II et aussi il montre le mécanisme d'importance méthodologique, des deux questions qui sont clés et qui aident aux chercheurs jeunes débutants à réfléchir sur le rôle qui jouent les cadres onto-épistémologiques et idéologiques dans la construction d'une nouvelle théorie. De la même façon l'article souligne I'usage critique et réflexif de la théorie tout au long du processus de la recherche ainsi que l'importance d'augmenter, rendre problématique et contraster le corpus théorique existent.
Palavras-chave descritor Epistemologia, teoria da educação, análise qualitativa.

\section{Resumo}

Neste artigo propõem-se e ilustram-se os conceitos de polo epistemológico I (relacionado ao marco teórico do pesquisador) e polo epistemológico II (relacionado à perspectiva dos participantes de uma realidade educativa). Propõe-se que o trânsito constante e permanente entre ambos os polos, bem como a aplicação do mecanismo de vigilância epistemológica permitem a cons trução de conhecimento teórico em pesquisa qualitativa. $O$ artigo finaliza dando especial atenção ao uso da teoria durante as diversas etapas do processo investigativo e às formas em que as teorizações iniciais podem ser descartadas ou ampliadas.

\section{Transferência à prática}

Este artigo refere-se a questões epistemológicas que são chave para a construção de novo conhecimento no âmbito educativo. Propõe e exemplifica os conceitos de polo epistemológico I e II, bem como destaca o mecanismo de relevância metodológi$\mathrm{ca}$, questões chave que ajudarão tanto a pesquisadores jovens como a seniors a refletirem sobre o papel dos marcos ontoespistêmicos e ideológicos na construção da nova teoria. Também realça a necessidade do uso crítico e reflexivo de teoria ao longo do processo investigativo, bem como a importância de aumentar, problematizar e contrastar o corpus teórico existente. 


\section{Introducción}

En los últimos diez años he llevado a cabo diversas investigaciones de corte cualitativo en el área educativa, cuyas configuraciones iniciales de los problemas de investigación han tenido diversos orígenes. Por un lado, la definición del problema de estudio lo he construido desde mi propio quehacer como académica universitaria, pero también con base en la lectura de grandes teorías en el ámbito sociológico, educativo y filosófico, así como de literatura especializada en el área.

Así, por ejemplo, realicé un estudio de caso de corte etnográfico acerca de la manera en que los profesores noveles aprenden a enseñar, como respuesta a la reflexión y problematización de sus prácticas pedagógicas y las mías propias (Guzmán-Valenzuela, 2009; 2012; 2013; Guzmán-Valenzuela \& Barnett, 2013a); he indagado por la construcción de identidades académicas en universidades chilenas, inspirada por estudios hechos en Reino Unido (Guzmán-Valenzuela \& Barnett, 2013b; 2013c) y en la actualidad estoy iniciando una investigación respecto al rol público de la universidad, motivada tanto por una discusión mundial acerca del papel de la universidad contemporánea como por la realidad de Chile, en la que lo público tiende a diluirse en un sistema universitario estratificado y mercantilizado. En todos estos casos y en aquellos en los he participado de modo más indirecto (mediante la supervisión de trabajos de investigación de estudiantes de pre y posgrado), he encontrado formulaciones de problemas de investigación híbridos, en el sentido en que se configuran en parte a partir de su enraizamiento en la práctica o el quehacer profesional y en parte por marcos teórico-disciplinares del investigador. Otras etapas del proceso investigativo, como la recolección y el análisis de los datos, también incorporan esta dualidad investigador-realidad estudiada. Desde mi experiencia, por ejemplo, mientras investigaba con mi equipo la construcción de identidades académicas, se produjo de manera constante un interjuego entre los marcos simbólicos y de interpretación de los investigadores y los contextos y significados de los participantes de una realidad educativa particular.

En este artículo analizo la relación entre el investigador y lo investigado cuando se llevan a cabo estudios en el campo educativo. Propondré que para la construcción de conocimiento acerca de la realidad educativa debe existir un tránsito continuo del investigador entre un polo epistemológico más cercano a los marcos interpretativos del investigador y otro más próximo a la visión de los participantes. Tal movimiento, además, es permanente, pues se produce durante todo el proceso investigativo y no solo durante la etapa de análisis de datos; ilustraré lo anterior con ejemplos provenientes de mi experiencia investigativa. Por último, relevaré el uso de la teoría para la búsqueda de nuevos marcos de comprensión de la realidad educativa.

\section{La dualidad sujeto cognoscente-objeto conocido y su interacción}

Hacer referencia a cuestiones epistemológicas en investigación conduce necesariamente a formularse preguntas acerca de la filosofía de la ciencia y las teorías del conocimiento. No es mi intención extenderme aquí en este tipo de cuestiones filosóficas, debido a que la complejidad y el grado de abstracción con los que se puede llegar a abordar ameritarían la escritura de varios artículos io libros! Más bien, me interesa entregar algunas definiciones simples que permitan, sobre todo al lector que se está iniciando en investigación, entender de qué estamos hablando cuando se utiliza el término "Epistemología".
Descripción del artículo | Article description | Description de l'article | Artigo descrição

Artículo de reflexión sobre dos investigaciones de corte cualitativo ya finalizadas: "¿Qué significa ser un académico universitario?: construcción de identidades en la universidad del siglo XXI" y "El proceso de construcción del conocimiento de oficio en profesores universitarios noveles: un estudio de casos en la Universitat de Barcelona". Ambas sirven de insumo para analizar cuestiones epistemológicas a la hora de llevar a cabo investigación educativa y la manera como se relaciona el investigador con aquello que es investigado, con énfasis en el mecanismo de vigilancia epistemológica. El artículo aborda el uso de la teoría en investigación a lo largo de sus diferentes fases y cómo puede ser ampliada, desafiada o descartada. 
En términos epistemológicos simples, en la construcción del conocimiento en investigación social y educativa existe una relación de la que participan el investigador que conoce y la realidad que está siendo conocida. Existe, por tanto, una dualidad sujeto-objeto o investigador-realidad investigada, en la que el primero no es reductible a la segunda y viceversa $y$, a la vez, un diálogo o una interacción entre ambos. Si seguimos los aportes del realismo social crítico (Archer, 1995; Bhaskar, 1998; Scott, 2010), considero relevante hacer la distinción entre el objeto de investigación como cosa real (realidad social que existe, con independencia de si un sujeto puede conocerla o no ${ }^{1}$ ) y el conocimiento que genera el sujeto cognoscente acerca de este, que puede coincidir por completo (en una situación ideal) o de manera parcial con el objeto como tal. Esta última posibilidad es la que, de acuerdo con mi visión, más representa el conocimiento humano, puesto que el conocedor construye teorías - ya sean científicas o de sentido común-acerca del objeto, que pueden acercarse más o menos a este y que, por tanto, son falibles, como propone el realismo social crítico (Scott, 2010).

Según el paradigma al que se adscribe el investigador educativo, la relación entre el investigador y la realidad estudiada puede ser de diferente naturaleza. Desde un paradigma positivista, el sujeto cognoscente se encuentra separado de la realidad (sea esta física o social) e intenta aprehenderla de manera objetiva, controlarla y predecirla. Existe una clara diferenciación entre sujeto y objeto y la interacción se produce en tanto el primero manipula y controla al segundo de manera escrupulosa e intenta no afectarlo para comprobar ciertas hipótesis previas. El sujeto cognoscente se mantiene distante del objeto conocido y guarda una posición de neutralidad y objetividad (Rosiek, 2013).

Desde un paradigma interpretativo (Howell, 2013; Savin-Baden, 2013; Rosiek, 2013; Vasilachis, 2006; Latorre, Del Rincón \& Arnal, 2003), abocado al estudio de la realidad social, la distinción entre sujeto que conoce (entendido como un sujeto que porta sus propios marcos de interpretación) y la realidad estudiada (concebida como un conjunto de significados que surgen de las interacciones en la vida social, en un grupo de personas, organizaciones educativas o sistemas educativos y sociales más amplios) (Denzin \& Lincoln, 2008), es más difusa. La relación entre sujeto cognoscente y realidad conocida se caracteriza por una interacción dinámica y constante en la que el sujeto, al conocer, afecta o modifica la realidad conocida y, a la vez, es afectado o modificado por esta. Existe, por tanto, una relación dialéctica, cuyo producto es un conocimiento co-construido que se va configurando a lo largo de las diversas etapas que constituyen el proceso de investigación. En el caso del paradigma denominado crítico (Rosiek, 2013; Howell, 2013; Savin-Baden, 2013; Latorre et al., 2003), no solo se produce este conocimiento co-construido - que, en general, surge en torno a un problema a solucionar-, sino que, además, los investigadores intervienen de modo activo para producir una transformación de las prácticas sociales y educativas, se convierten en conocedores y participantes a la vez (sujeto y objeto de investigación) y producen modificaciones en la realidad social (que deja de ser lo que era²).

1 Para más detalles del realismo social crítico, sugiero leer el artículo de David Scott contenido en esta edición especial.

2 La investigación cualitativa en Educación suele adscribirse a uno de estos dos paradigmas o a una combinación de ambos: el interpretativo o el crítico-transformativo. También existen posicionamientos más eclécticos o pragmáticos, en los que la vinculación a uno(s) de estos paradigmas no es evidente porque se utilizan métodos mixtos de investigación. En el artículo de David Scott contenido en este monográfico se analiza este tema desde una perspectiva ontoepistémica. 
En este artículo me interesa profundizar en la comprensión de la relación dinámica que se genera entre los marcos de comprensión propios del investigador y aquellos más cercanos a los participantes de una realidad educativa en una investigación de tipo cualitativo. En esta dirección apunta la distinción que se hace entre perspectivas etic/emic en investigación cualitativa que discutiré en la siguiente sección.

\section{Perspectivas etic y emic: más allá de la interpretación}

La distinción entre perspectivas emic/etic se ha utilizado para dar cuenta del proceso de interpretación, es decir, el proceso a partir del cual se otorga sentido a los datos (Howell, 2013; Huberman \& Miles, 1994). La perspectiva etic se refiere a las descripciones acerca de una realidad desde un punto de vista externo a ella (la del investigador); en cambio, la perspectiva emic se trata de la visión que tienen los sujetos sobre una realidad cultural determinada de la cual participan (Pérez, 1998).

Las expresiones "investigar acerca de la realidad" e "investigar desde la realidad" podrían mostrar la distinción etic/emic; investigar "acerca de" la realidad denota una posición más lejana o externa a la realidad e investigar "desde" indica una más cercana. En el primer caso, el investigador hará descripciones y elaborará interpretaciones más alejadas de la experiencia de los participantes (aunque siempre en diálogo con esta) y conectadas con marcos interpretativos y ontoepistémicos afines a su disciplina, lo cual permite que estas interpretaciones sean más abstractas. En el segundo caso, el investigador estará "más atento" a las visiones y los significados de los participantes, con especial atención a sus prácticas sociales y diálogos cotidianos en contextos singulares de donde emergen significados creados y negociados e intenciones que organizan y revelan la experiencia como un proceso (Huberman \& Miles, 1994). Para lograrlo, el investigador realizará descripciones densas (Geertz, 1973), con cuidado de los detalles, la complejidad y los significados situados en la vida diaria, privilegiará una estancia prolongada en el campo y/o analizará y validará los resultados del estudio con los propios participantes.

En esta misma línea, algunos autores (Navarrete, 2011; Díaz, 2009; Huberman \& Miles, 1994) proponen que el análisis inicial de los datos daría origen a una dimensión descriptiva o inductiva del mismo, también denominada de primer orden (perspectiva emic) y la síntesis posterior en el que estos datos se integran a categorías deductivas conectadas con teorías más abstractas originarían una dimensión de segundo orden (perspectiva etic). Como resultado de este juego dialéctico que Giddens y Dallmayr (1982) Ilaman doble hermenéutica, se origina la teoría o la construcción de conocimiento en investigación cualitativa.

Aquí me permito hacer dos afirmaciones: en primer lugar, tanto el análisis de primer orden o descriptivo (perspectiva emic) como el de segundo orden (perspectiva etic) son interpretativos. El investigador no solo asigna significados a las acciones, interacciones e interpretaciones de los participantes (Rosiek, 2013; Savin-Baden, 2013; Huberman \& Miles, 1994; Schwandt, 1994) cuando utiliza determinadas teorías disciplinares, sino también cuando organiza y relaciona la información que recoge, de unas determinadas maneras $^{3}$. Así, por ejemplo, cuando el investigador cualitativo decide dar a conocer o describir ciertos diálogos o interacciones y no otros, ya está haciendo una selección de los relatos o significados, cuestión que obedece a intereses del investigador (Erickson, 1989). En otras palabras, las teorías implícitas del investigador actúan como marco para dar sentido a los datos y determinar cuáles son relevantes y cuáles no. En consecuencia, si bien la interpretación como proceso puede encontrarse más o menos cercana a la perspectiva de los participantes (emic) o a la de teorías de un(os) campo(s) disciplinar(es) (etic) —en este último caso, el análisis es más teórico o abstracto-, ambos tipos de análisis son interpretativos. En segundo lugar, como propone Giddens y Dallmayr (1982), la doble hermenéutica involucra un juego dialéctico entre las dimensiones más inductivas o descriptivas (perspectiva emic) y las más deductivas o teóricas (perspectiva etic); es simultáneo y constante y, por momentos, puede estar más cerca de la perspectiva emic o etic. Aún más, propongo que el constante movimiento que hace el investigador entre las perspectivas etic y emic se encuentra presente en todo el proceso investigativo y no solo en la etapa de análisis de datos, cuestión que discutiré en las próximas secciones.

\section{Etapas del proceso investigativo y polos epistemológicos}

Lo dicho hasta aquí nos muestra que:

1 Durante las diversas etapas del proceso investigativo $-\mathrm{y}$ no solo durante el proceso de análisis e interpretación de los datos-es

3 Una propuesta similar realiza Ashwin (2012) cuando plantea que, en el proceso de construcción de un objeto, el investigador utiliza tanto un lenguaje interno de descripción como uno externo. En principio, esta distinción fue propuesta por Basil Bernstein para diferenciar entre una descripción basada en modelos conceptuales (lenguaje interno de descripción), de una descripción sistemática y estructurada que proviene de la evidencia empírica (lenguaje externo de descripción). En ambos casos, la descripción va más allá del dato duro. 
posible diferenciar entre dos extremos que llamaré polos epistemológicos I y II. El polo epistemológico I ubica al investigador en una postura más alejada en su relación con la realidad estudiada y más cercana a la teoría. Por teoría entiendo los grandes marcos disciplinares, ontológicos (formas de concebir la realidad) y epistemológicos (formas de relacionarse con la realidad) del investigador, así como las ideologías de base y los posicionamientos valóricos. El polo epistemológico II posiciona al investigador más cerca de las visiones e interpretaciones de los participantes de una realidad determinada e incluso podría formar parte de ella 4 . Estos polos quedarían representados por las perspectivas etic y emic, respectivamente, aunque, como señalé, ambos están presentes en todo el proceso de investigación y no solo en la etapa de interpretación de los datos (Figura 1).

Figura 1

Polos epistemológicos | y ||

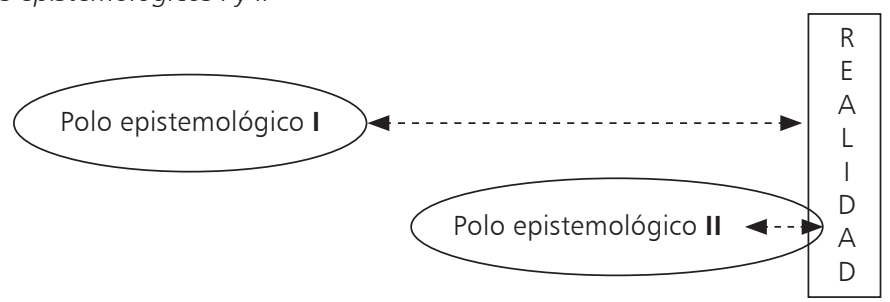

Fuente: elaboración propia

2 Los polos epistemológicos no son mutuamente excluyentes, sino que forman parte de un continuo, de manera que el investigador cualitativo no se ubica en uno u otro; más bien, se mueve entre ambos durante las etapas de la investigación (Figura 2). Como resultado de este movimiento constante a lo largo del proceso investigativo entre polos epistemológicos y, por ende, de mayor o menor cercanía a la realidad estudiada, se produce un conocimiento que incorpora una síntesis de la perspectiva del investigador y sus marcos teóricos y la perspectiva de los participantes (Howell, 2013; Rosiek, 2013; Schwandt, 1994; Guba \& Lincoln, 1994).

Figura 2.

Tránsito continuo del investigador entre los polos epistemológicos I y |l durante el proceso investigativo

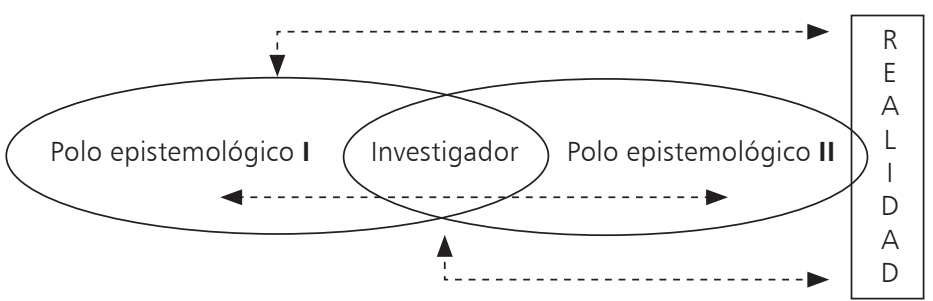

Fuente: elaboración propia

4 Este sería el caso de investigaciones que se inscriben en el denominado paradigma transformativo-crítico en el que el investigador es ambos: sujeto conocedor y parte de la realidad investigada. También corresponde a la investigación etnográfica, en la que el investigador forma parte de la realidad estudiada, aunque no pretende transformarla. 
A continuación ilustraré el tránsito del investigador cualitativo entre los polos epistemológicos I y II durante las etapas de construcción del problema de investigación, la formulación del marco teórico y la recolección y análisis de los datos ${ }^{5}$, con atención al uso de teorías disciplinares. Es importante recalcar que hago esta distinción de etapas con fines descriptivos y analíticos, pues en investigación cualitativa estas fases suelen solaparse (Latorre et al., 2003; Sandín, 2003).

\section{Uso de la teoría durante el proceso de investigar: ¿Antes, durante o después?}

Algunas de las discusiones que aparecen en la literatura proponen el rol de la teoría durante el análisis y la interpretación de los datos. Teóricos de la Grounded Theory debaten si es posible o no prescindir de la teoría a la hora de iniciar una investigación (Thornberg, 2011; Thomas \& James, 2006). Sin embargo, dichos debates giran en torno a la decisión de usar o no teoría al inicio de la investigación o a cómo utilizarla en etapas finales del estudio.

Mi interés aquí es examinar el uso de la teoría en investigación educativa según la nomenclatura de polos epistemológicos que he propuesto, durante diversas etapas del proceso investigativo. Así, al analizar el movimiento continuo del investigador entre los polos epistemológicos I y II emerge, por ejemplo, la pregunta sobre qué viene primero y después al inicio de una investigación: ¿El investigador define un problema de estudio desde el polo epistemológico II y luego se mueve al polo epistemológico I o viceversa? Asimismo, surgen cuestionamientos acerca de cómo se acoplan la teoría con los datos empíricos en la recolección y el análisis de datos.

\section{Formulación del problema de investigación}

En la Introducción aludí al hecho de que mis propias prácticas pedagógicas y los dilemas sobre cómo enseñar me llevaron a plantearme un problema de investigación que decidí explorar de manera sistemática mediante un estudio de caso de corte etnográfico. Por tanto, la formulación inicial del problema de investigación tuvo su origen en un dilema que yo misma enfrentaba como investigadora y que deseaba comprender o profundizar. La definición del problema de investigación se ubicó en una posición más cercana a la realidad, esto es, en el polo epistemológico II (Figura 3).

Figura 3.

Definición inicial del problema de investigación desde el polo epistemológico II

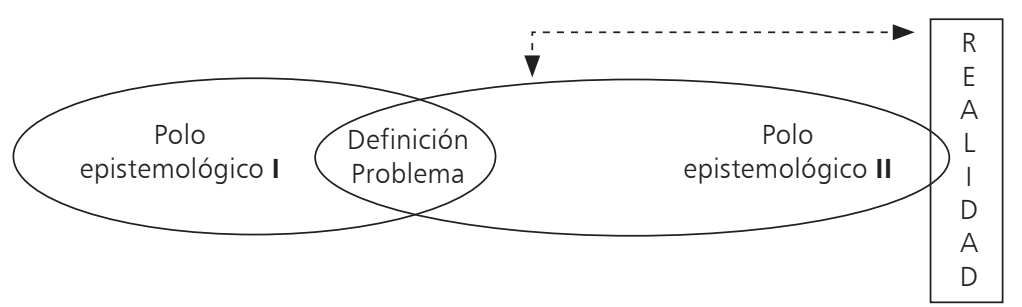

Fuente: elaboración propia

5 La cuestión del análisis de datos y su interpretación será abordada de manera detallada por José Luis Medina en el artículo que incluye esta edición especial. 
Esta postura también queda ilustrada cuando se utilizan estrategias de investigación del tipo investigación-acción participativa, en la que existe un investigador externo que actúa como facilitador (Kemmis, McTaggart \& Nixon, 2014; Rosiek, 2013; Latorre et al., 2003) o el Design Based Research (Van den Akker, Gravemeijer, McKenney \& Nieveen, 2006) ${ }^{6}$. En ambas situaciones el rol del investigador externo consiste en facilitar la definición de un problema que experimentan los participantes de una realidad educativa y ayudar en la formulación y evaluación de intervenciones con el fin de darle solución. El gran desafío del investigador externo es dejar espacio y protagonismo a los participantes para que sean ellos mismos quienes intervengan de manera activa en la formulación de un problema práctico y para que, con ayuda del investigador, planifiquen, intervengan y evalúen una intervención. El investigador debe estar atento y vigilante para no intentar dar respuestas absolutas a los problemas o conducir el estudio de acuerdo con sus propios intereses disciplinares y marcos conceptuales (polo epistemológico I).

También puede presentarse el caso de que un investigador formule un problema a ser estudiado que no necesariamente provenga del quehacer o de la práctica. Este problema de investigación queda delimitado por el investigador que, a partir de la literatura, considera que el estudio de un problema está inconcluso o abre nuevas preguntas para su investigación. La definición del problema de investigación es más bien de tipo teórico y su construcción queda sujeta a unos determinados marcos de conocimiento disciplinar que el investigador utiliza para abordar un problema (Figura 4). La formulación del problema de investigación queda supeditada a los marcos conceptuales y ontoepistémicos que utiliza el investigador, los cuales pueden ser variados y dar origen a diversas preguntas de investigación. Hay una primacía de la perspectiva del investigador en la formulación inicial del problema de estudio.

Figura 4.

Definición del problema de investigación desde el polo epistemológico I

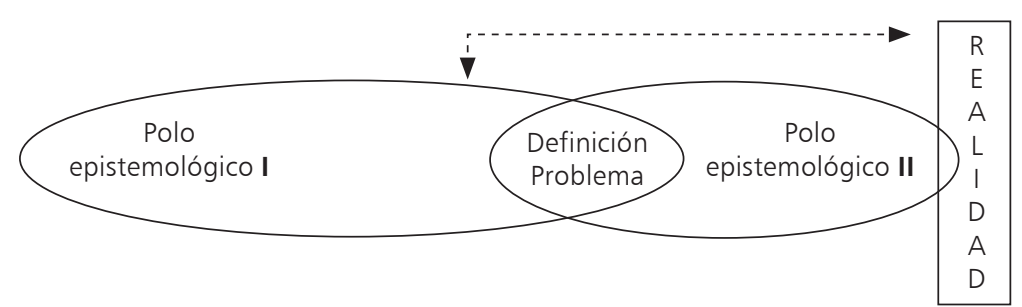

Fuente: elaboración propia

Antes de pasar a la siguiente sección quiero hacer dos acotaciones importantes: en primer lugar, el planteamiento acerca de que la formulación de un problema de investigación se ubica más cerca a la teoría o a las visiones e interpretaciones de los participantes — polos epistemológicos I y II, respectivamente- no tiene connotaciones evaluativas (en el sentido de que una sea mejor que la otra). En segundo lugar, la distinción entre ambas posturas no implica que sean incompatibles o inmutables; por lo contrario, la formulación inicial de un problema podría verse modificada en

6 Para ampliar información puede verse el artículo de Marta Romero contenido en esta edición especial. 
el transcurso de la investigación en la medida en que se ganen nuevas comprensiones del objeto de estudio proporcionadas, ya sea por nuevos marcos interpretativos hallados o por las visiones de los participantes y las características de su contexto.

\section{¿Con o sin marco teórico?}

El uso de marcos teóricos en investigación ha sido discutida en diversos artículos en los que se analiza el desarrollo teórico de la investigación educativa en educación superior (Tight, 2004; 2007; Ashwin, 2012). En ellos se concluye que en Estados Unidos y algunos países de Europa y Asia hay un alto porcentaje de artículos publicados que son ateóricos (en Estados Unidos, alrededor de un $25 \%$ y en otros países, cerca de un 50\%) (Tight, 2004, 2007). En cuanto a los artículos en los que los autores explicitan los marcos teóricos desde donde se posicionan, estos marcos teóricos pueden ser de distinto tipo (Goetz \& LeCompte, 1988). Por un lado, existen teorías amplias, robustas y explicativas (grandes teorías sociológicas o filosóficas en las que se puede apreciar un posicionamiento ontológico y epistemológico) y, por otro, existen teorías intermedias, es decir, un conjunto de proposiciones que intentan explicar de manera abstracta un comportamiento social. El uso de teorías intermedias en ocasiones parece obedecer más a modas que a una decisión fundada en una discusión crítica (puesto que determinado marco conceptual es más apropiado para abordar un problema que otro) (Ashwin, 2012) ${ }^{7}$. Por último, también se ha encontrado que en el apartado de marco teórico algunos artículos dan cuenta de una diversidad de perspectivas teóricas sin que el autor se identifique con alguna de ellas (Ashwin, 2012).

La cuestión de la elaboración de un marco teórico y de un estado del arte en torno a un problema de investigación es importante para los investigadores que se están iniciando o para aquellos profesionales del ámbito educativo que están sumidos en las prácticas y tienen poco "contacto" con la producción de conocimiento en el campo educativo desarrollado en los últimos años. De acuerdo con mi experiencia, los profesionales y estudiantes en el área son hábiles en la identificación de una situación problemática en tanto esté ligada a sus prácticas profesionales cotidianas; sin embargo, cuando intentan justificar la relevancia y pertinencia de investigar un problema, muchas de sus aseveraciones se enraízan en nociones de sentido co-

7 La Grounded Theory propone otra distinción entre teorías: la teoría sustantiva, que emerge a partir de la captura y el análisis sistemático de los datos y la teoría formal, que se refiere a una teoría más abstracta y universal asociada con disciplinas científicas, a la que se llega mediante el método de las comparaciones constantes (Glaser \& Strauss, 1967) mún y en cuestiones muy concretas provenientes de la práctica. También tienden a ignorar importantes teorías, constructos y métodos para estudiarlos que han sido bastante utilizados en el mundo educativo y que podrían servir para abordar la problemática detectada. Tras reconocer la relevancia de la utilización de un marco conceptual alrededor de un problema de investigación, surgen algunos interrogantes y dilemas acerca de su uso en investigación cualitativa en Educación.

Una primera aplicación de la teoría en investigación educativa puede ser de tipo tautológico. En este caso, la investigación - que se vuelve tendenciosa"encuentra" lo que, en términos teóricos, se pensaba encontrar de antemano. Por tanto, la teoría determina los resultados 0 , para ser más precisa, genera unos resultados que se acomodan a los marcos teóricos previos. Como señala Ashwin (2012), el ejercicio empírico de recoger datos es más bien de tipo ilustrativo (porque los datos ilustran una teoría, no la ponen a prueba), sin mayor análisis crítico o expansión de dicha teoría. En otras palabras, el proceso de investigación se utiliza con fines confirmatorios. En consecuencia, todo el proceso investigativo - la formulación del problema de investigación, la recolección de datos, su análisis e interpretación - se construyen en torno a unos marcos conceptuales que tienden a ser confirmados con el examen de los datos. No existe una mayor indagación acerca de qué tan adecuada es esa teoría para comprender un problema educativo o si podría existir alguna otra teoría que permitiera revisarlo de una manera diferente.

Para continuar con la terminología de polos epistemológicos que he propuesto, diré que, en el caso del uso tautológico de la teoría, el investigador se ubicaría en el polo epistemológico I con una finalidad autoconfirmatoria de la teoría y casi sin moverse hacia el polo epistemológico II a lo largo del proceso investigativo (Figura 4). Así, las visiones e interpretaciones de los participantes (polo epistemológico II) se acomodarían a la teoría del investigador. Este tipo de aproximación en investigación educativa no promovería el desarrollo teórico (Ashwin, 2012; Anyon, 2008) ${ }^{8}$.

Un mecanismo que ayudaría al investigador a salvaguardar esta situación guarda relación con el concepto de vigilancia epistemológica (Bourdieu, Chamboredon y Passeron, 2002; Willig, 2001), que hace referencia a un proceso continuo de autorreflexión por parte de los investigadores en relación con sus propias posturas intelectuales y sus marcos ontológicos,

8 Algo similar ocurre cuando se llevan a cabo investigaciones bajo ciertas constricciones financieras o valóricas que conducen a "crear" aquellos resultados que son convenientes para unos determinados fines. Para un análisis crítico de estas cuestiones, pueden verse los artículos de Torrego y de Barnett y Parry, en esta edición especial. 
epistemológicos e ideológicos ${ }^{9}$ y de qué manera estos condicionan y obstaculizan su aproximación al estudio de un problema de investigación. Lo anterior implica tomar distancia del conocimiento de sentido común y romper con las preconcepciones respecto a un objeto de estudio para construir un nuevo conocimiento. El examen constante de estas perspectivas intelectuales permite mantener la coherencia entre la teoría empleada y el método de investigación, así como analizar si la teoría se adecúa o no al segundo y qué limitaciones presenta (Blanco, 2010). A la vez, este ejercicio involucra ser "sensible a los datos" (Denzin \& Lincoln, 2008), es decir, acercarse a las visiones de los participantes e intentar reconstruirlas a partir de su contexto. La vigilancia epistemológica, entonces, no es más que la expresión del ejercicio sistemático y reflexivo de movimiento constante entre los polos epistemológicos I y || (Figura 5):

Figura 5.

Vigilancia epistemológica durante el tránsito entre los polos epistemológicos I y II

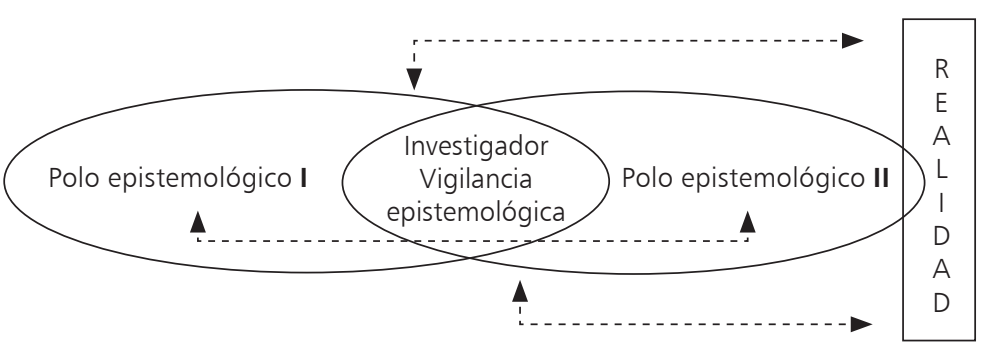

Fuente: elaboración propia

Para volver a la pregunta sobre si iniciar investigación con o sin la construcción de un marco teórico, me interesa discutir algunas posibilidades y posicionarme al respecto. Mi postura es muy cercana a los planteamientos de Thornberg (2011) y Thomas y James (2006), quienes justifican el uso de la teoría desde el inicio de la investigación y critican a algunos adeptos de la Grounded Theory, que insisten en retrasar el uso de la teoría hasta etapas finales. Mi visión es que, para un investigador con cierta experiencia y trayectoria, esto es imposible. Sus marcos de conocimiento están conformados por una serie de teorías del propio campo disciplinar y afines, así como de premisas ontoepistémicas. En tal sentido, no sería factible acercarse a la realidad sin marcos interpretativos, aunque sí existiría la capacidad del investigador de hacer un ejercicio para explicitarlos y convertirlos en objeto de su reflexión crítica (vigilancia epistemológica). En el caso de investigadores noveles, si bien podrían carecer aún de marcos teórico-conceptuales robustos, sí cuentan con teorías de sentido común (conformadas por experiencias, creencias y conocimiento adquirido a lo largo de la vida escolar y académica) que guían la manera de interactuar con la realidad. De nuevo la vigilancia epistemológica, como mecanismo, resulta de utilidad para explicitar el rol de los marcos ontoepistémicos e ideológicos del investigador en la creación de nueva teoría.

9 El ejercicio reflexivo en torno al posicionamiento ideológico me parece fundamental en Ciencias Sociales y Educación, en especial cuando se estudian temáticas educativas delicadas, como las relacionadas con uso y abuso de poder en diversos ámbitos, discriminación y cuestiones religiosas o cuando existen intereses creados al iniciar una investigación que obedecen a cuestiones políticas o económicas. 


\section{Recolección y análisis de datos y tránsito por los polos epistemológicos}

En este apartado quisiera referirme a algunas experiencias de investigación durante las etapas de recolección y análisis de datos —etapas que suelen solaparse en investigación cualitativa- que permiten ilustrar el continuo movimiento entre los polos epistemológicos I y II y que, a la vez, representaron desafíos o situaciones dilemáticas.

La primera experiencia guarda relación con un estudio que inicié hace un par de años con mi equipo respecto a identidad académica en universidades chilenas (Guzmán, 2011). Esta es una temática poco explorada en el mundo iberoamericano $y$, en particular, en Chile. Señalo esto porque la idea de elaborar el proyecto surgió de una estancia académica que realicé en el Oxford Learning Institute entre los años 2010 y 2011, en donde conocí la temática del trabajo académico muy estudiada en Reino Unido y en otros países anglosajones. Mi primera aproximación conceptual a esta temática en ese tiempo fue bastante "fragmentada", en el sentido de que tuve acceso a una serie de artículos de revistas y ponencias que no explicitaban del todo los marcos interpretativos desde donde se posicionaba el investigador. Más bien, se planteaban ciertas teorías intermedias o definiciones en torno a la identidad o al trabajo académico y se hacía referencia, sobre todo, a cuestiones disciplinares, de género y experiencia que podrían impactar en las formas de construir la identidad académica (Clegg, 2008; Henkel, 2005; Välimaa, 1998; Becher \& Trowler, 2001).

Cuando decidí formular mi propio proyecto de investigación en Chile, mi marco teórico se caracterizó por un conjunto de teorías intermedias acerca de la identidad académica, provenientes de la Psicología Social, la descripción de estudios llevados a cabo en países anglosajones, una breve contextualización del sistema universitario chileno y la justificación de la relevancia del estudio, en tanto no existían investigaciones en esta área en este país. Con base en los planteamientos de Ashwin (2012), podría decir que el marco teórico de mi proyecto de investigación incluía en sus inicios microteorizaciones de moda en un contexto ajeno al mío más que grandes teorías filosóficas o sociológicas en torno a la temática y con las cuales yo, como investigadora, no me posicionaba mayormente.

Durante el desarrollo del estudio y antes de comenzar la recolección de los datos, una nueva visita al país inglés, cuyo objetivo consistió en "apropiarme conceptualmente" de la temática en estudio, me permitió acceder a otros marcos de comprensión a la hora de estudiar identidad académica, uno de tipo psicológico y otro sociológico. Si bien mi formación inicial es en Psicología, pronto deseché esta aproximación, pues mi intención era analizar el fenómeno de la identidad académica sin centrarme en el sujeto; más bien, deseaba estudiarlo en el contexto particular chileno y opté por una visión sociológica, con prioridad de un análisis socioestructural. Por recomendación de colegas expertos en el área, leí a la autora Margaret Archer (1995; 1996; 2000; 2003; 2007), lo que constituyó todo un descubrimiento para mí. Yo estaba familiarizada con las teorías bourdieuranas hasta cierto punto; sin embargo, los planteamientos archerianos abrieron una ventana de comprensión del interjuego entre las grandes macroestructuras sociales y la agencia ${ }^{10}$.

Debo reconocer que los textos de esta socióloga contemporánea no son fáciles de leer (más aún cuando solo uno de sus libros está traducido al castellano), así que la lectura fue lenta y pausada y me encontraba en este proceso cuando ya estaba recogiendo los datos del proyecto con mi equipo. Lo anterior significó que, si bien las primeras entrevistas no fueron afectadas por este marco conceptual, sucedió que, en la medida en que yo misma y uno de mis ayudantes de investigación leíamos y discutíamos la teoría archeriana, esta iba "tiñendo" irremediablemente tanto las siguientes entrevistas que efectuamos como su revisión. Durante el análisis de datos, hicimos el ejercicio de delimitar con claridad las categorías inductivas (más apegadas a los datos y, por lo tanto, al polo epistemológico II) frente a las categorías deductivas (polo epistemológico I) asociadas con los postulados de Archer. En este último caso, justificábamos conceptualmente de qué manera la teoría se adecuaba o no a los datos recogidos tras la discusión y reflexión constante entre miembros del equipo investigador, es decir, mediante una triangulación de perspectivas del investigador que ponía en acción el mecanismo de vigilancia epistemológica ${ }^{11}$.

Dos cuestiones más que quiero discutir antes de finalizar esta sección guardan relación con la investigación sobre identidades académicas mencionada, estudio que motivó en mí la necesidad de reflexionar en torno a cuestiones epistemológicas en investigación cualitativa en Educación. Ambas cuestiones son de tipo dilemático, en tanto no parecían tener una solución que se encontrara en un manual de investigación cualitativa. La primera de ellas está ligada con mi rol como investigadora en un estudio en el que exploraba

10 Algo similar sucedió con una colega de una universidad irlandesa (O'Byrne, 2013) quien, en un seminario acerca de investigación y perspectivas archerianas, señaló que "encontró" este marco conceptual mucho más tarde, cuando ya había recogido los datos de su estudio. En términos metodológicos, esta colega sugirió que su análisis deductivo de las categorías había sido retroactivo, pues la teoría que dio sentido a los datos apareció tras la recogida.

11 Para más detalles acerca del análisis de identidad académica a la luz de la teoría archeriana, puede verse Guzmán-Valenzuela \& Barnett (2013a). 
la cuestión de la identidad docente, mientras era académica de una universidad chilena. En las entrevistas individuales, no fue un ejercicio fácil dejar que el entrevistado pudiera expresar sus propias visiones con libertad y fluidez, sin intentar conducir el diálogo hacia aquellas temáticas y problemáticas que a mí misma, como académica, me llamaban la atención o me cuestionaban. Para evitar esta dificultad, antes de comenzar la recolección de datos, mi equipo y yo creamos un guion de entrevista semiestructurada, que permitiera a los entrevistados abordar las temáticas clave del estudio de una manera abierta y con la menor cantidad de interrupciones y dirección del relato. Otra acción útil fue la constante discusión que tuvo el equipo en torno a los datos y que activaron el mecanismo de vigilancia epistemológica. Por otro lado, la posibilidad de escribir un artículo sobre identidades académicas en Chile y la experiencia del tiempo (Guzmán-Valenzuela \& Barnett, 2013b) y la necesaria lectura adicional que debí hacer me permitieron darme cuenta de que yo no era la única que enfrentaba este tipo de dificultades (Ylijoki \& Mäntylä, 2003); por lo contrario, parece ser un dilema comúnmente enfrentado, pero pocas veces explicitado en la literatura especializada.

La segunda cuestión guarda relación con el proceso de análisis de datos y la aparición de conceptos que no estaban contemplados en la construcción teórica inicial del objeto de estudio y que emergieron de manera reiterada en el discurso de los participantes del estudio sobre identidad académica. En el caso de este estudio, durante las entrevistas y los grupos focales apareció con frecuencia la problemática del tiempo en la vida académica, a pesar de que esta cuestión no estaba incluida en los objetivos de investigación. Sin embargo, parecía ser un asunto transversal e inmanente a las experiencias de ser académico, pues cada vez que se hablaba de las tareas de docencia, investigación gestión y administración o de los límites entre la vida privada y la personal, la cuestión del tiempo resurgía. Por tanto, al inicio del análisis de datos, identificamos numerosos fragmentos que codificamos de manera inductiva, con el propio lenguaje de los participantes. A continuación, mi decisión como investigadora fue leer literatura contemporánea que me permitiera "iluminar" estos pasajes inductivos. Así, acudí a conceptualizaciones propuestas por Adam (1995), Barnett (2011) e Ylijoki (2010; 2013), entre otras, para proponer "categorías dialécticas de análisis" (Guzmán-Valenzuela \& Barnett, 2013b). El nombre de categorías dialécticas fue sugerido por el profesor José Luis Medina, de la Universitat de Barcelona, a quien, mediante una comunicación personal, le expuse la dificultad en torno a cómo denominar categorías que eran tanto inductivas (sensibles) como deductivas en su naturaleza.
Ambos dilemas presentados y su solución ilustran el continuo movimiento entre los polos epistemológicos I y II, tanto en la etapa de recolección como de análisis de los datos; además, dan cuenta de cómo se pone en marcha el mecanismo de la vigilancia epistemológica de manera sistemática e intencionada.

\section{Conclusiones}

En este artículo he propuesto varias cuestiones de orden epistemológico que bien pueden ser de utilidad no solo para la investigación cualitativa en el ámbito educativo, sino para la investigación cualitativa en general. Sobresalen la distinción de polos epistemológicos y el tránsito continuo y permanente del investigador entre ambos en todo el proceso de investigación cualitativa, así como el uso del mecanismo de vigilancia epistemológica para velar que haya un movimiento constante entre ambos polos de manera metódica y regular.

También me he referido al papel de la teoría durante el proceso investigativo. En la literatura sobre investigación cualitativa es posible encontrar una gran variedad de trabajos dedicados a la discusión en torno a la necesidad de construir teoría a partir de los datos o lo que se denomina teoría fundada (Denzin \& Lincoln, 2008; Eisenhardt \& Graebner, 2007), que ha tenido gran impacto en la forma de hacer investigación. Esta discusión resulta esencial en investigación cualitativa, pues por medio de ella se quieren rescatar los significados que los propios participantes asignan a una realidad de la que participan. Se trata de que el investigador nunca pierda de vista esa realidad; al contrario, debe utilizar todos los mecanismos posibles para no perder de vista la perspectiva de los participantes y su contexto. Asimismo, ha habido discusión sobre la importancia de que el investigador esté atento a sus marcos ontoepistémicos y sus posicionamientos ideológicos a la hora de construir un objeto de estudio (Medina, 2005). No obstante, resulta menos evidente, al menos en la región iberoamericana, el debate sobre el rol de la teoría en la investigación cualitativa.

Con ejemplos provenientes de mi experiencia investigativa en el ámbito educativo, he querido señalar que el uso de la teoría está presente en todo el proceso investigativo y no solo cuando se construye un marco conceptual en torno a un problema de investigación o cuando se está en la etapa de análisis interpretativo de los datos. Esta presencia es deseable, en tanto la finalidad de la investigación cualitativa no radica en comprender una realidad, sino también en aumentar y contrastar su corpus teórico. Para lograrlo, resulta crucial conocer el estado del arte de un problema de investigación para discutirlo, desafiarlo, ampliarlo o desecharlo, en caso de que no sea útil. Creo que este 
es un ejercicio que ha quedado un tanto olvidado, al dar primacía a una actitud autoindulgente y poco rigurosa en investigación que tiende a confirmar o ilustrar teorías, más que a problematizarlas o deconstruirlas.

El investigador cualitativo debe estar preparado para hacer un ejercicio crítico-reflexivo que le permita construir nuevas y mejores teorías para comprender la realidad. Con ese fin, debe privilegiar tanto la teoría que se construye desde los datos y los participantes, como aquella que proviene de autores y colegas del área disciplinar y afines. Este constante interjuego que por momentos puede crear tensiones y dilemas en investigación cualitativa- es necesario y saludable en el oficio de crear nuevo conocimiento en Educación; si no, ¿con qué otro fin nos embarcaríamos en el desafío de investigar?

\section{Sobre la autora}

Carolina Guzmán-Valenzuela ha investigado durante los últimos diez años sobre las prácticas de enseñanza en diferentes niveles educativos, con especial énfasis en educación superior. En la actualidad indaga acerca del trabajo académico y el rol de la universidad en el siglo XXI.

\section{Referencias}

Adam, B. (1995). Timewatch: The Social Analysis of Time. Cambridge: Polity Press.

Anyon, J. (2008). Theory and Educational Research: Toward Critical Social Explanation. Nueva York: Routledge.

Archer, M. (1995). Realist Social Theory: The Morphogenetic Approach. Cambridge: Cambridge University Press.

Archer, M. (1996). Culture and Agency: The Place of Culture in Social Theory. Cambridge: Cambridge University Press.

Archer, M. (2000). Being Human. The Problem of Agency. Cambridge: Cambridge University Press.

Archer, M. (2003). Structure, Agency and the Internal Conversation. Cambridge: Cambridge University Press.

Archer, M. (2007). Making our Way through the World. Human Reflexivity and Social Mobility. Cambridge: Cambridge University Press.

Ashwin, P. (2012). How Often are Theories Developed through Empirical Research into Higher Education? Studies in Higher Education, 37(8), 941-955.

Barnett, R. (2011). Being a University. Londres: Routledge. Becher, T., \& Trowler, P. (2001). Academic Tribes and Territories: Intellectual Enquiry and the Culture of Disciplines. Filadelfia: Open University Press.

Bhaskar, R. (1998). General Introduction. En Archer, M., Bhaskar, R., Collier, A., Lawson, T., \& Norris,
A. (eds.). Critical Realism: Essential Readings. Londres y Nueva York: Routledge.

Blanco, C. (2010). La vigilancia epistemológica en Ciencias Sociales: un compromiso ineludible. Reflexiones desde la sociología del conocimiento de Pierre Bourdieu. Recuperado de http://www.expoesia. com/media/Ponencia_Blanco_Cecilia.pdf

Bourdieu, P., Chamboredon, J., \& Passeron, J. (2002). El oficio del sociólogo. Presupuestos epistemológicos. Buenos Aires: Siglo XXI.

Clegg, S. (2008). Academic Identities under Threat? British Educational Research Journal, 34(3), 329-345.

Denzin, N., \& Lincoln, Y. (2008). The Landscape of Qualitative Research. (3a ed.). Londres: Sage Publications.

Díaz, C. (2009). ¿Cómo desarrollar, de una manera comprensiva, el análisis cualitativo de los datos? Educere, 13(44), 55-66.

Eisenhardt, K., \& Graebner, M. (2007). Theory Building from Cases: Opportunities and Challenges. Academy of Management Journal, 50(1), 25-32.

Erickson, F. (1989). Métodos cualitativos de investigación sobre la enseñanza. En Wittrock, M. (comp.). La investigación en la enseñanza II. Métodos cualitativos y de observación. Madrid: Paidós.

Geertz, C. (1973). The Interpretation of Cultures: Selected Essays. Nueva York: Basic Books.

Giddens, A., \& Dallmayr, F. R. (1982). Profiles and Critiques in Social Theory. Berkeley: University of California Press.

Glaser, B. G., \& Strauss, A. L. (1967). The Discovery of Grounded Theory: Strategies for Qualitative Research. Chicago: Aldine Publishing Company.

Goetz, J. P., \& LeCompte, M. D. (1988). Etnografía y diseño cualitativos en investigación educativa. Madrid: Morata.

Guba, E., \& Lincoln, Y. (1994). Competing Paradigms in Qualitative Research. En Denzin, N., \& Lincoln, Y. (eds.). Handbook of Qualitative Research. Thousand Oaks: SAGE Publications.

Guzmán-Valenzuela, C. (2009). Developing Craft Knowledge in Teaching at University: How do Beginning Teachers Learn to Teach. European Educational Research Journal, 8(2), 326-335.

Guzmán-Valenzuela, C. (2011). ¿Qué significa ser un académico universitario?: construcción de identidades en la universidad del siglo XXI. (Investigación inédita). Fondecyt, Santiago.

Guzmán-Valenzuela, C. (2012). Enseñanza reflexiva y profesores universitarios noveles: desafíos para la mejora de la enseñanza. Revista de la Educación Superior, XLI(3), 115-137.

Guzmán-Valenzuela, C. (2013). Challenging Frameworks for Understanding Teaching Practices in Higher Education: The End or the Beginning? Qualitative Research in Education, 2(1), 65-91. 
Guzmán-Valenzuela, C., \& Barnett, R. (2013a). Academic Fragilities in a Marketised Age: The Case of Chile. British Journal of Educational Studies, 61(2), 1-18.

Guzmán-Valenzuela, C., \& Barnett, R. (2013b). Marketing Time: Evolving Timescapes in Academia. Studies in Higher Education, 38(8), 1120-1134.

Guzmán-Valenzuela, C., \& Barnett, R. (2013c). Developing Self-Understanding in Pedagogical Stances: Making Explicit the Implicit Among New Lecturers. Educação e Pesquisa, 39(4), 891-906.

Henkel, M. (2005). Academic Identity and Autonomy in a Changing Policy Environment. Higher Education, 49(1-2), 155-176.

Howell, C. (2013). Qualitive Research. En Savin-Baden, C., \& Howell, C. Qualitative Research: The Essential Guide to Theory and Practice. Londres: Routledge.

Huberman, M., \& Miles, M. (1994). Data Management and Analysis Methods. En Denzin, N., \& Lincoln, Y. (eds.). Handbook of Qualitative Research. Thousand Oaks: SAGE Publications.

Kemmis, S., McTaggart, R., \& Nixon, R. (2014). The Action Research Planner: Doing Critical Participatory Action Research. Nueva York: Springer.

Latorre, A., Del Rincón, D., \& Arnal, J. (2003). Bases metodológicas de la investigación educativa. Barcelona: Experiencia.

Medina, J. L. (2005). Deseo de cuidar y voluntad de poder: la enseñanza de la enfermería. Publicacions i Edicions Universitat de Barcelona.

Navarrete, J. (2011). Problemas centrales del análisis de datos cualitativos. Revista Latinoamericana de Metodología de la Investigación Social, 1(1), 47-60.

O'Byrne, C. (2013). Academic Lives through a Lens: Exploring and Explaining the Formation of Academic Professional Identities Using Archer's Theories [presentación en PowerPoint]. Recuperado de http://www.srhe.ac.uk/downloads/ events/75_C_O_Byrne.ppt

Rosiek, J. (2013). Philosophical Stance. En Savin-Baden, C., \& Howell, C. Qualitative Research: The Essential Guide to Theory and Practice. Londres: Routledge.
Sandín, M. P. (2003). Investigación cualitativa en Educación: fundamentos y tradiciones. Madrid: McGraw Hill.

Savin-Baden, M. (2013). A Brief History of Philosophical Perspectives. En Savin-Baden, C., \& Howell, C. Qualitative Research: The Essential Guide to Theory and Practice. Londres: Routledge.

Schwandt, T. (1994). Constructivist, Interpretivist Approaches to Human Inquiry. En Denzin, N., \& Lincoln, Y. (eds.). Handbook of Qualitative Research. Thousand Oaks: SAGE Publications.

Scott, D. (2010). Education, Epistemology and Critical Realism. Londres: Routledge.

Thomas, G., \& James, D. (2006). Reinventing Grounded Theory: Some Questions about Theory, Ground and Discovery. British Educational Research Journal, 32(6), 767-795.

Thornberg, R. (2011). Informed Grounded Theory. Scandinavian Journal of Educational Research, 56(3), 243-259.

Tight, M. (2004). Research Into Higher Education: An A-Theoretical Community of Practice? Higher Education Research and Development, 23(4), 395-411.

Tight, M. (2007). Bridging the Divide: A Comparative Analysis of Articles in Higher Education Journals Published Inside and Outside North America. Higher Education, (53), 235-253.

Van den Akker, J., Gravemeijer, K., McKenney S., \& Nieveen N. (eds.) (2006). Educational Design Research. Londres: Routledge.

Vasilachis de Gialdino, I. (2006). Estrategias de investigación cualitativa. Barcelona: Gedisa, 280.

Willig, C. (2001). Introducing Qualitative Research in Psychology: Adventures in Theory and Method. Filadelfia: Open University Press.

Ylijoki, O-H. (2010). Future Orientations in Episodic Labour: Short-Term Academics as a Case in Point. Time \& Society, 19(3), 365-386.

Ylijoki, O-H. (2013). Boundary Work between Work and Life in the High-Speed University. Studies in Higher Education, 38(2), 242-255.

Ylijoki, O-H., \& Mäntylä, H. (2003). Conflicting Time Perspectives in Academic Work. Time \& Society, 12(1), 55-78. 